\title{
First results from THEMIS spectropolarimetric mode ${ }^{\star}$
}

\author{
A. López Ariste, J. Rayrole, and M. Semel \\ DASOP, URA 326, Observatoire de Paris, Section de Meudon, 92190 Meudon, France
}

Received June 29; accepted October 12, 1999

\begin{abstract}
We present here the very first spectropolarimetric results obtained with the multiline spectroscopy mode (MTR) of THEMIS. The principal problems found during the data analysis are exposed. A first characterisation of the quality of this observing mode is given.
\end{abstract}

Key words: astronomical instrumentation: telescopes astronomical techniques: polarimetric — astronomical techniques: spectroscopy

\section{Introduction}

The French-Italian solar telescope THEMIS (Téléscope Heliographique pour l'Étude du Magnetisme et des Instabilités Solaires) opened its first observational campaign with polarimetric capabilities last summer. In this paper we present the results of the first reduction of data coming from the MTR polarimetry mode. Spectropolarimetric observations are at the origin of the special design of this telescope. Two main aspects characterize it: first, the telescope is free from systematic instrumental polarization, apart from some residuals, mainly expected at the entrance and exit windows. Second, it allows observation of up to 10 spectral domains simultaneously. These two characteristics distinguish THEMIS from any other solar telescope built to date.

The interest of a polarization-free telescope is selfevident. Nevertheless from the very impressive results obtained by ASP at the VTT at Sacramento Peak (Martínez Pillet et al. 1997; Westendorp Plaza et al. 1997) or ZIMPOL at the MacMath Telescope at Kitt Peak (Stenflo \& Keller 1996) using telescope calibration, a polarization-free instrument avoids all the hard work this calibration involves (Skumanich et al. 1997). It will, in the future, constitute an advantage for seeking higher

\footnotetext{
^ Based on observations made with THEMIS operated on the island of Tenerife by CNRS-CNR in the Spanish Observatorio del Teide of the Instituto de Astrofísica de Canarias.
}

polarimetric sensitivities, which require analysis of the smallest instrumental residuals. With respect to the second characteristic, a lot of work has been devoted to the insufficiency of a single spectral line for a correct determination of magnetic field (and of the atmosphere parameters in general) as a function of depth (Rayrole 1967; Rayrole 1971; Semel 1981; del Toro Iniesta et al. 1990). There is a necessity of measuring simultaneously several sensitive lines in order to determine the magnetic field and its gradients along the line of sight unambiguously (as well as other atmospheric quantities). This is one of the multiple advantages that any multi-wavelength observation provides, even if limited to the visible (and neighbouring) spectrum, as in the case of THEMIS. Hence, this telescope appears as a promising instrument for the measurement of magnetic fields in the solar photosphere, the purpose of our observations.

THEMIS was, during this observing campaign, on the fine-tunning phase of its instruments. Our observations therefore provided much more information on what was happening in the telescope than on solar phenomena. Furthermore although many of the remaining problems should be solved for next campaigns, we consider that this first data reduction from the MTR mode of THEMIS still constitutes a good description of how the instrument behaves, how the data should be handled and what can be expected in the near future from this observing mode.

A brief description of our observations is made in next section. Sections 3 and 4 form the core of this work: the data reduction techniques are presented, the main defects are described, and several measures of observed noise are shown. Section 5 is devoted to illustrate the kind of data that the THEMIS MTR mode provides after reduction, together with preliminary longitudinal magnetic field charts.

\section{Observations}

The observations were made in August 1998, between the 18th and the 24th. The telescope, a Ritchey-Chrétien for which an optical scheme is supplied in Figs. 1 and 2, 
Table 1. Possible polarization observing modes as a function of the position of the 2 quarter-wave plates. Plate \# 1 is the nearest to the beam splitter

\begin{tabular}{c|c|r} 
Plate \# 1 & Plate \# 2 & Measurement \\
\hline 0 & 0 & $I \pm Q$ \\
0 & 22.5 & $I \pm\left(\frac{1}{2} Q-\frac{\sqrt{2}}{2} V+\frac{1}{2} U\right)$ \\
0 & 45 & $I \mp V$ \\
\hline 22.5 & 0 & $I \pm\left(\frac{1}{2} Q+\frac{\sqrt{2}}{2} U+\frac{1}{2} V\right)$ \\
22.5 & 22.5 & $I \pm U$ \\
22.5 & 45 & $I \pm\left(\frac{1}{2} U-\frac{\sqrt{2}}{2} Q-\frac{1}{2} V\right)$ \\
\hline 45 & 0 & $I \pm U$ \\
45 & 22.5 & $I \pm\left(\frac{\sqrt{2}}{2} U-\frac{\sqrt{2}}{2} Q\right)$ \\
45 & 45 & $I \mp Q$
\end{tabular}

operated in the so-called MTR mode (Mein \& Rayrole 1985; Rayrole \& Mein 1993). This mode allows polarimetric observations of the sun in up to 10 different spectral regions simultaneously. Two CCDs are available for each spectral region (see Fig. 2) to record the two orthogonal polarization signals provided by the polarization analyzer, which was situated in the Cassegrain focus of the telescope. At this position little systematic instrumental polarization is introduced (during the observations, the cooling system for the entrance and exit windows was not operating, hence an amount of instrumental polarization can be expected due to thermal stress in the glass). The analyzer consists of a fixed calcite beam splitter and two rotating quarter-wave plates (see Fig. 2). Three positions were available for each one of the plates: $0,22.5^{\circ}$ and $45^{\circ}$ relative to a privileged direction given by the optical axis of the calcites. This setup allowed successive observation of $I \pm Q, I \pm U$ and $I \pm V$ (see Table 1) but it did not allow path interchange for the two signs of each polarization. Therefore, after the calcite, one of the optical paths was always assigned to the $I+$ Stokes signals, and the other one to the $I$-Stokes ones. A first wide slit was placed just before the analyzer. Opened to 12 arcsec, it limited the field of view of the observations to $12^{\prime \prime} \times 120^{\prime \prime}$. After the transfer optics, the two paths reached the second focus where the true slit was placed, opened to 0.5 arcsec. From there it entered the spectrograph. A mask after the predispersing grating isolated the spectral regions where the selected lines were situated. At the exit of the spectrograph were placed the 4 pairs of CCD cameras used in these observations. Each pair observed one spectral region in $I+$ Stokes and $I$-Stokes respectively. All the regions for each Stokes parameter are taken strictly simultaneously in this setup. The observed lines and the spectral dispersion in the region covered by the CCDs around them are indicated in Table 2.

After examination of the data, we have concentrated our efforts on the analysis of the observations of August $22 \mathrm{nd}$. In spite of a consistent Sahara dust-driving wind, at
Table 2. List of solar lines observed, and the spectral resolution obtained for each domain

\begin{tabular}{l|c|c} 
& date & Dispersion $(\AA /$ pixel $)$ \\
\hline Fe I 6301 $\AA$ and 6302 $\AA$ & 22-VIII & 0.0195 \\
Fe I 6149 $\AA$ and 6151 $\AA$ & 22 -VIII & 0.0185 \\
Fe I 5247.0 and 5247.5 & 22-VIII & 0.0065 \\
NaD1 5890 & 22-VIII & 0.0155 \\
Fe I 5576 & 23-VIII & 0.0166
\end{tabular}

that date several technical problems of the telescope had been solved and an almost complete series of observations and flat field images was available. All the scans covered a sunspot of the active region NOAA-8307. Each scan consisted of between 70 and 120 steps of 1 arcsec each. The scan was done by the telescope positioning system (a mirror is scheduled to be installed which will perform this and other tasks, but it was not available yet at the moment of the observations). For each step of the scan three exposures were taken to obtain in sequence $I \pm Q, I \pm U$ and $I \pm V$. Each exposure spanned $300 \mathrm{~ms}$. We stress the fact that all the 4 spectral domains and the two polarities were taken simultaneously. The readout of the 8 CCDs and the positioning of the analyzer for the following measure added up to $1 \mathrm{~s}$ per polarization and up to $3 \mathrm{~s}$ per scan step. This waiting time will be reduced in the future since some processes can be done in parallel (while they were done sequentially during our observations) and the CCD's readout accelerated by a judicious choice of windows.

A set of data of the spectral region around $5576 \AA$ comes from observations taken on August 23rd. This line is insensitive to magnetic field. It constitutes therefore a very interesting tool for the measure of the noise levels in polarization and for any polarization introduced by the instrument. Unfortunately this line was not observed simultaneously with the other spectral domains.

A series of images was taken around noon by moving the telescope in an elliptical path around the solar disc center and taking several hundred exposures. The flat field image (FFI) resulted from the combination of those images. Each one of the images still presents a residual of solar granulation, but the mean of all them is free of solar details (up to a residual of $0.6 \%$ of the intensity of the continuum), and it has been used for the reduction of the observations of the whole day as described in Sect. 3.2.

\section{Reduction techniques}

We intend in this section to describe the techniques used for the reduction of the data and to provide a first description of the observational status of THEMIS. The reduction procedures can be divided into two groups: geometrical corrections and flat field calibration, both 

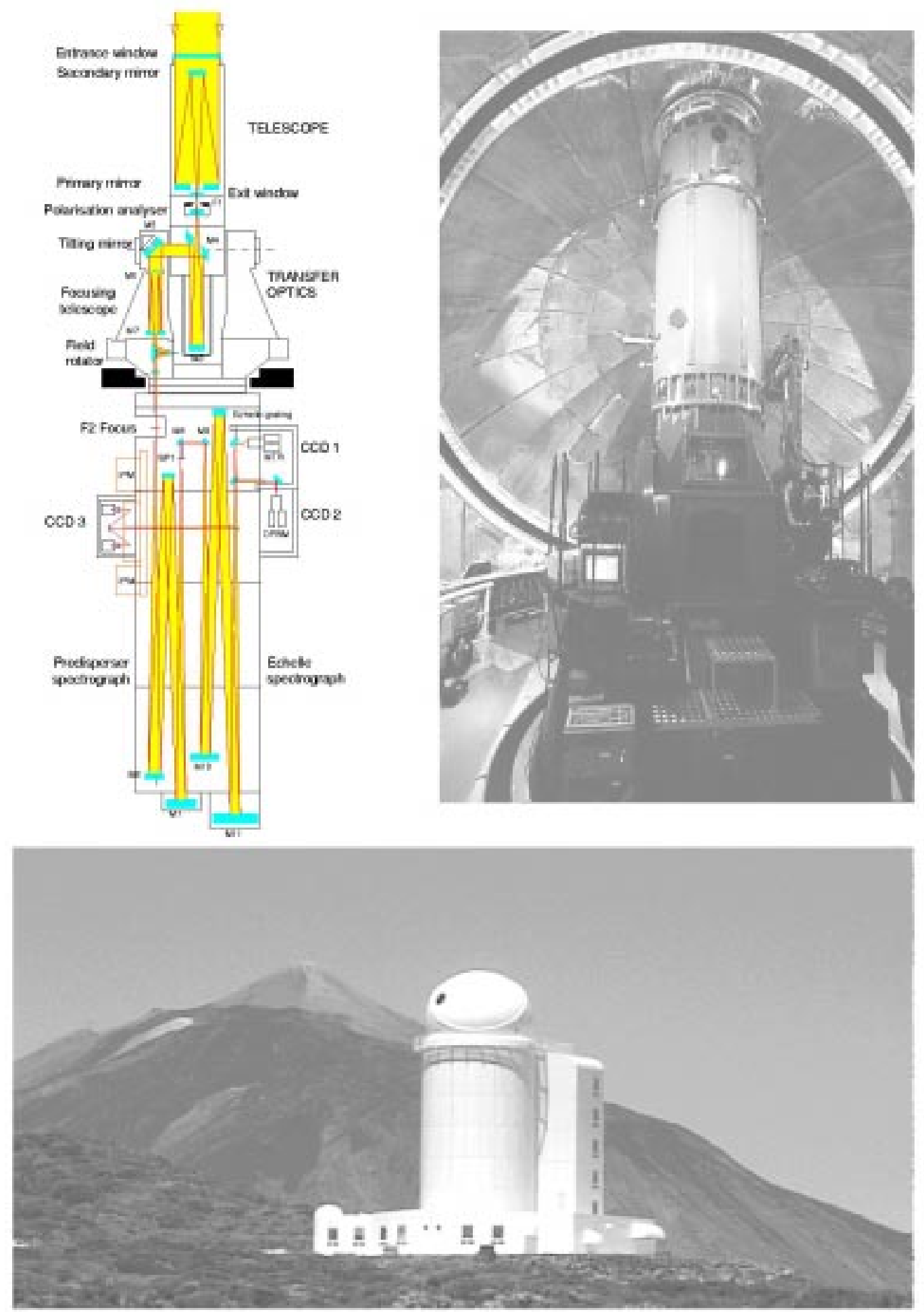

Fig. 1. Optical design of the MTR mode at the THEMIS telescope. Note the position of the polarization analyser, minimizing the instrumental polarization. Only one set of CCDs (CCD1) from the MTR mode is shown in the figure. Up to 10 pairs can work simultaneously, although only 4 were used in the present observing run. At top left is a picture showing the telescope in the dome, and the bottom image shows a general view of the THEMIS telescope 

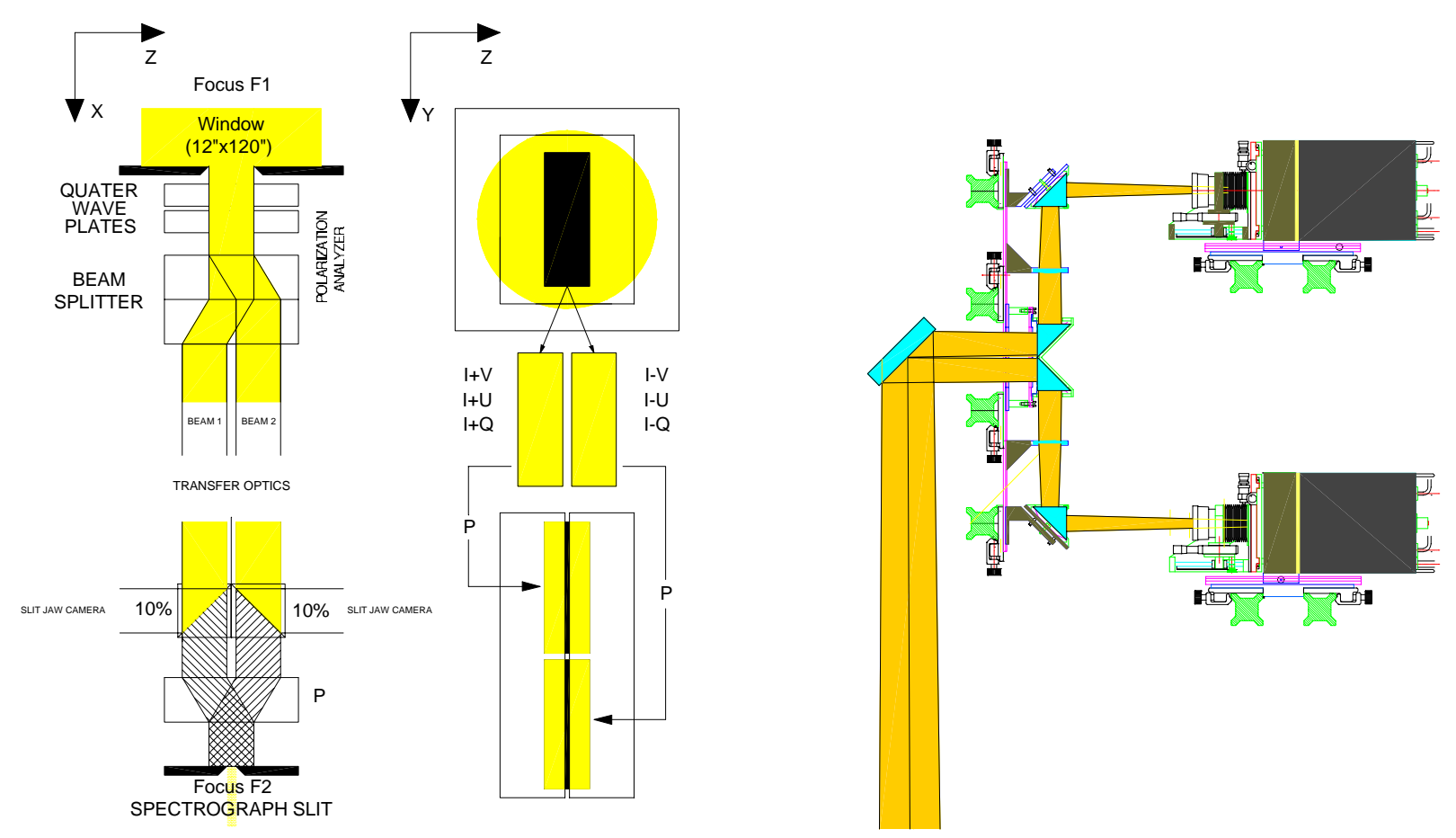

Fig. 2. Left: Polarization analyser diagram (the light comes from above). After the beam splitter the two images with orthogonal polarization are placed side by side. An optical system in the focus F2 is used to put them one above the other, as sketched in the central figure. Right: Close-up of the exit of the MTR mode with the 2 CCD cameras, one per path. This configuration is repeated for each spectral domain

necessary for the Stokes profiles reduction. This classification is done based on the assumed origin of the observed effects upon the images.

\subsection{Geometry corrections}

\subsubsection{Inclination and curvature corrections}

The first and most conspicuous geometry correction is the spectral redressing. Due to the off-axis configuration of the spectrograph, the iso-wavelength lines draw a 2nd order curve in the focal plane where the CCDs are placed. The images of the slit follow these curves (see Fig. 3). The dispersion direction is still parallel to the CCD's pixel rows (horizontal in the figure), but each row has suffered a shearing which must now be corrected by a displacement applied to each of them separately.

The analytical form of the curve can be calculated using coefficients that can be fitted from the flat-field data. But we can also rely on statistical methods which provide directly the shearing needed for each line of pixels. In this second approach we consider that in the FFI all the profiles along the slit are of the same shape, but displaced by an indeterminated quantity. We took as a reference the first row of the CCD and applied a Principal Component Analysis (PCA) technique to generate a base of the space of displaced profiles (Rees et al. 1999). Every other profile along the slit was compared against this database and a correction displacement determined within a precision of 0.01 pixels. To assess the limitations of both methods (the statistical and the fitting of a 2 nd order function) we compared the two orthogonally-polarised images of the Fe I line at $5576 \AA$. For this line, insensitive to magnetic fields, both images should be strictly identical except for global displacements of wavelength or flat field errors. Each image was redressed independently and compared to the other one, line by line, to see the difference in the calculated displacement. We did that for all the 314 component images of the FFI. In Fig. 4 we show the statistics of these comparisons. In the standard deviation graph we see a constant value for all the flats of about 0.03 pixels, which we can take as the error in the determination of these displacements.

The origin of this error is unclear. The PCA and the fitting methods have been tested with analytical profiles with extraordinary results, so we consider that the 0.03 pixels limit is mainly due to differences of the profiles along the slit, differences due probably to instrumental effects. For instance, the same line in both CCD's does not correspond necessarily to strictly the same zone in the sun. Therefore, slightly different radial velocities can affect the 

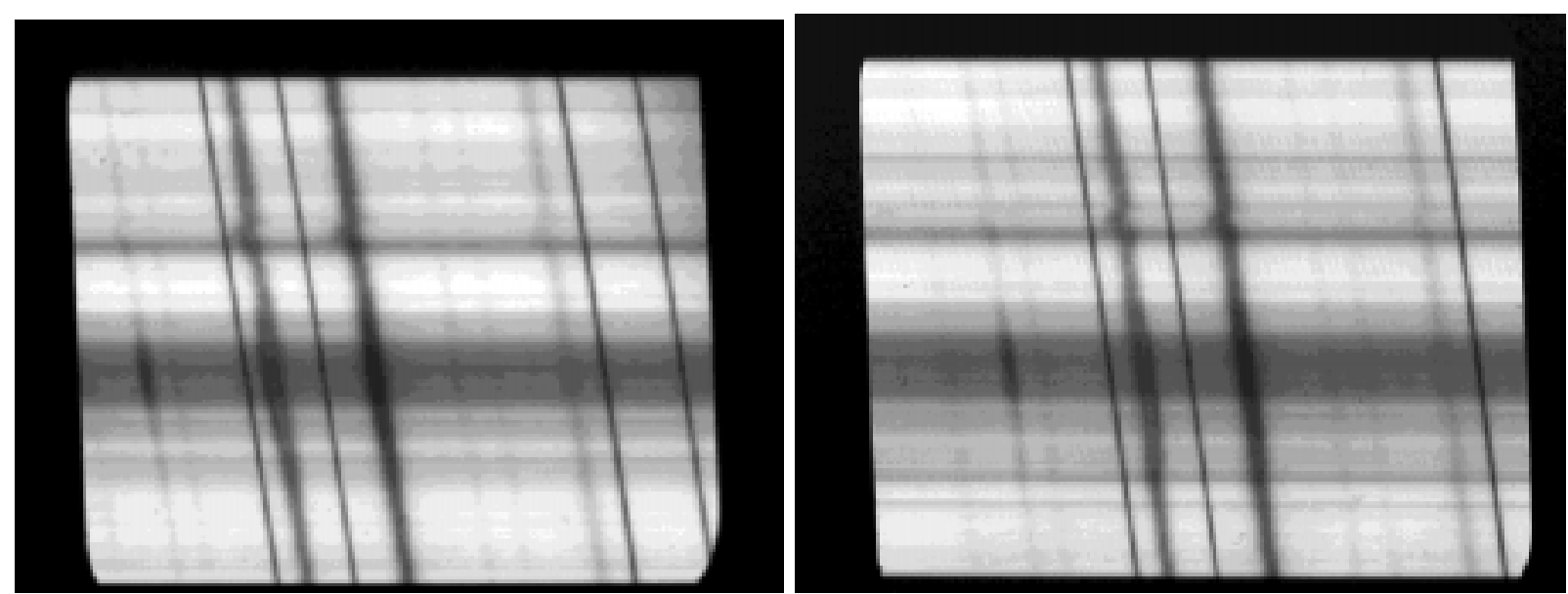

Fig. 3. Raw data for the Fe I 6301 and $6302 \AA$ lines showing line inclination due to the off-axis spectrograph setup. $I+V$ is shown at left and $I-V$ at right
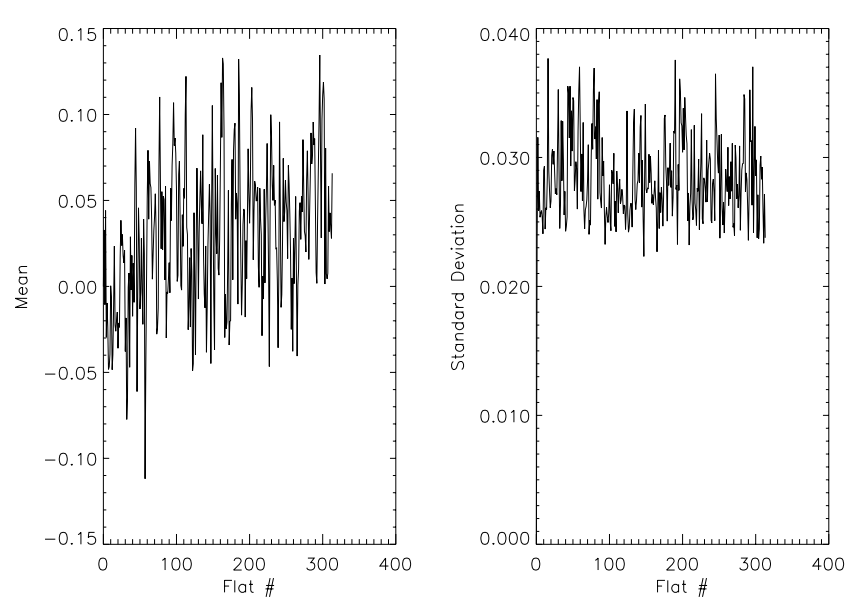

Fig. 4. Statistics of the difference of displacements between the two orthogonally-polarised images for a series of 314 component images of the FFI for the $5576 \AA$ Fe I line. Note the net displacement error of one path relative to the other due to an error in the superposition of the two first rows of each one of the comparison images

two profiles and a different displacement is determined for them.

\subsubsection{Dilatations and spatial corrections}

Indeed, one of the main problems of this first set of spectropolarimetric data from THEMIS has been the diagnostic of this spatial displacement. For the correct determination of the polarimetric signal, the orthogonallypolarized paths must be compared in exactly the same point of the sun. To ensure this, we have used the solar details as seen in the continuum by the two cameras. A correlation was established between them and a displacement was calculated. This method was sufficient whenever the solar details were highly contrasted, as in the presence of a spot, for instance. But with just granulation some errors appeared here and there which perturbed the measurements. This method must therefore be substituted or, at least improved by adding some other spatial references, such as for instance slit hairs or the limits of the field of view.

Slit hairs would also help in the future for the other severe problem in the determination and correction of the geometry of the two paths: the different optical reduction for the two cameras. One objective is used in front of each CCD to reduce the desired field of view to the size of the CCD chip. These objectives must be identically positioned for the two paths. In case they are not, a dilatation coefficient between both images must be applied. This dilatation is the same in both directions: spatial and spectral. The method used to measure and correct this dilatation has been the comparison of two separate spectral zones, inside the range covered by the camera, to their counterparts from the other camera. The effect of the dilatation was to produce an apparent displacement of one zone relative to the other. This displacement was used to calculate the dilatation coefficient. The results of this method depend on the spectral region. For instance we obtained very good corrections for the $6149-6151 \AA$ domain. A small window was defined around each one of the two lines and was compared to equally defined windows in the other camera. The $2 \AA$ which separate the two lines resulted in a high precision in the measurement of the coefficient of dilatation. In the case of the $6301-6302 \AA$ domain, the separation of just $1 \AA$ appeared to be in the limit of the method.

\subsection{Flat field calibration}

The flat field image (FFI) was calculated as described in the previous section. The FFI showed absence of any 
solar details, however the pseudo-random movement around the disk center (with a radius of about half the solar disk radius) had the undesired effect of widening the profiles. Nevertheless a mean profile was obtained from the FFI, useful to determine the necessary geometrical corrections. Also from the FFI we obtained a correction matrix in which all the defects of the optical path were apparent. Between these effects special attention must be paid to fringes. Two systems of interference fringes appeared in one of the paths. This asymmetry between the two paths served to identify some of the optical pieces that cause these fringes. One of these systems shows a low frequency ( $\sim 75$ pixels in period) and the correction matrix sufficed to remove it from the observations. The second one, on the other hand, with a much higher frequency ( $\sim 3$ pixels in period) was not removed by this correction. Probably, small displacements due to real image displacements, to errors in the determination of the superposition of the correction matrix and each one of the observations, or to movements of the system of fringes are the origin of this problem. A small difference in the position of the fringes in the correction matrix from that of the observations, coupled with the high frequency of these fringes, makes full correction impossible. For these first observations and data reduction, we used a simple filtering in the Fourier space, although a better solution must be found for the future.

Wavelength-dependent scattered light was found throughout the observing run. Most of this light came from the F1 focus environment which was not well isolated from the spectrograph, allowing all the light rejected by the slit to enter it as scattered light. This effect was the origin of one of the toughest problems encountered during these observations. Figure 5 shows the equivalent widths of the Fe I line at $5576 \AA$ for the two paths and for all the Stokes signals. There is a clear difference between them of about $10 \%$. The 3 polarisations for each path give similar values and the observed difference is much bigger than the errors in the determination of the equivalent width. This difference between the two paths results in a spurious signal in the subtracted image which strongly limits the polarimetric sensitivity ${ }^{1}$.

\section{Polarimetric sensitivity and noise estimations}

Several tests have been performed to assess the noise in the data. We have measured three different types of noise. None of them includes systematic errors. We anticipate that with an improved setup those errors will disappear, or at least will be greatly diminished in the future. They have been discussed in previous sections: differences in the equivalent widths for each path, etc. The noise discussed

\footnotetext{
${ }^{1}$ Our last observations with THEMIS in August 1999 show
} that this problem seems to be solved.

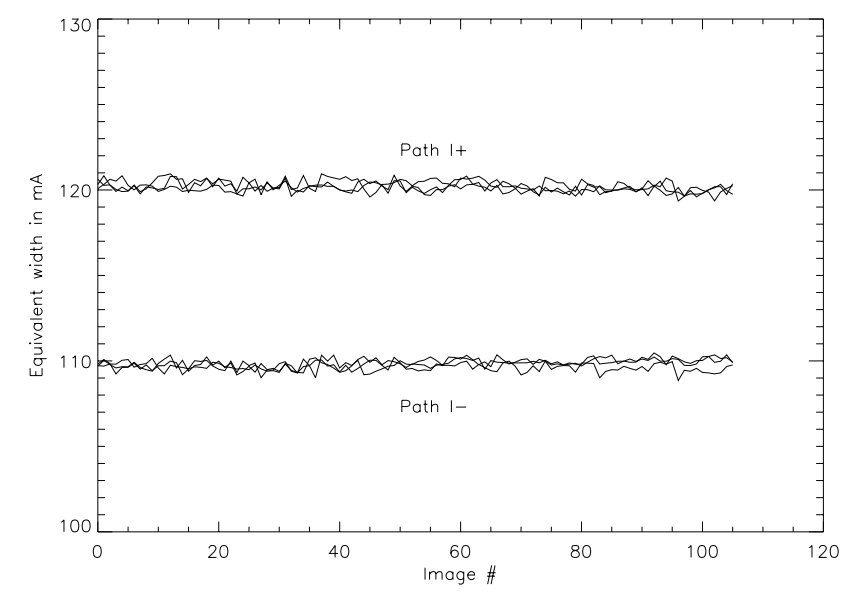

Fig. 5. Measured equivalent width (in $\mathrm{m} \AA$ ) of the Fe I $5576 \AA$ line for the two paths and all the analyzer positions $(I \pm Q, I \pm U$ and $I \pm V)$ in a series of 100 images of solar disk center on August 23rd

here is that which remains if those systematic errors were not present.

\subsection{Photon noise}

We have measured the photon noise per pixel using the continuum region around the Fe I line at $5576 \AA$ (data obtained on August 23rd, see Table 1). One hundred images of the disk centre for the Stokes $V$ parameter were obtained at around 7:50 UT. The best way to measure photon noise is to compare two images identical except for the noise. As an approximation to this ideal we compared each image with the next one. But solar surface details were visible in the images, therefore we created two mean images by adding the odd and even numbered images respectively. The final images are free from solar features and comparison is done between the nearest images possible. A mean profile of the selected continuum region was taken for each mean image by adding the profiles along the slit. At this point, each one of the mean profiles contains $I+\delta_{i}(i=1,2)$ : a common signal $I$ and an added noise $\delta_{i}$. The ratio of the two profiles gave us (neglecting second order terms):

$\frac{I+\delta_{1}}{I+\delta_{2}}=1-\frac{\delta_{2}-\delta_{1}}{I}$.

The mean value of this ratio is therefore 1 if the noise is well distributed around zero. The standard deviation of the ratio corrected for the number of images and the number of profiles along the slit (used to generate the mean profile) gives the noise per pixel. The resulting measured value is $\sigma=2.410^{-3}$. This value is consistent with a constant of $250 \mathrm{e}^{-} / \mathrm{ADU}$ (Analog-Digital Unit), as defined by the CCD's chipset constructor. 

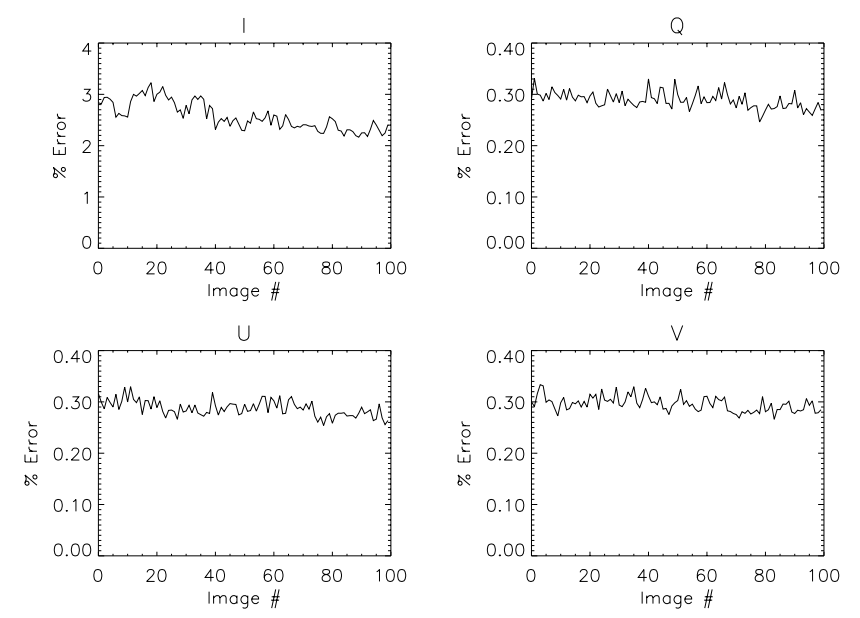

Fig. 6. Standard deviations measured in the continuum around the Fe I line at $5576 \AA$ in the Stokes signals from a series of images of the disk center. The $3 \%$ of Stokes $I$ is due to residual solar surface granulation (the telescope was randomly displaced during the exposures). The values in the other Stokes parameters are an indication of the photon noise and the errors accumulated in the data reduction process

\subsection{Polarimetric noise in the continuum}

Next, we searched for the error in the continuum region in the reduced images of Stokes $I, Q, U$ and $V$. The same 100 images of the disk center at $5576 \AA$ were used for this purpose, but after reducing them as explained in previous sections and obtaining the Stokes parameters by simple addition and substraction. Each set of profiles from the four resulting images for the Stokes parameters was interpreted as a sole statistical variable. No consideration was made about how they had been obtained. The continuum in $Q, U, V$ should show a null signal plus an error. In practice we observed a mean signal of $210^{-4}$ indicating a problem with the normalization of the continuum signal from the two paths. The standard deviation of each one of the images in the selected region of the continuum is shown in Fig. 6. In Stokes $I$ the obtained value of around $3 \%$ is due to residual solar granulation(the telescope was randomly displaced during the exposures). Excepting this, the other 3 Stokes parameters show a common standard deviation for the whole series of images of approximately $310^{-3}$ of the intensity of the continuum. This noise is slightly higher than the measured photon noise. For an exposure time of $300 \mathrm{~ms}$, this number gives the polarimetric sensitivity of the instrument at the moment of the observations (August 1998).

\subsection{Polarimetric noise in the lines}

The previous measurement was done in the continuum in the disk center and near a line not sensitive to the

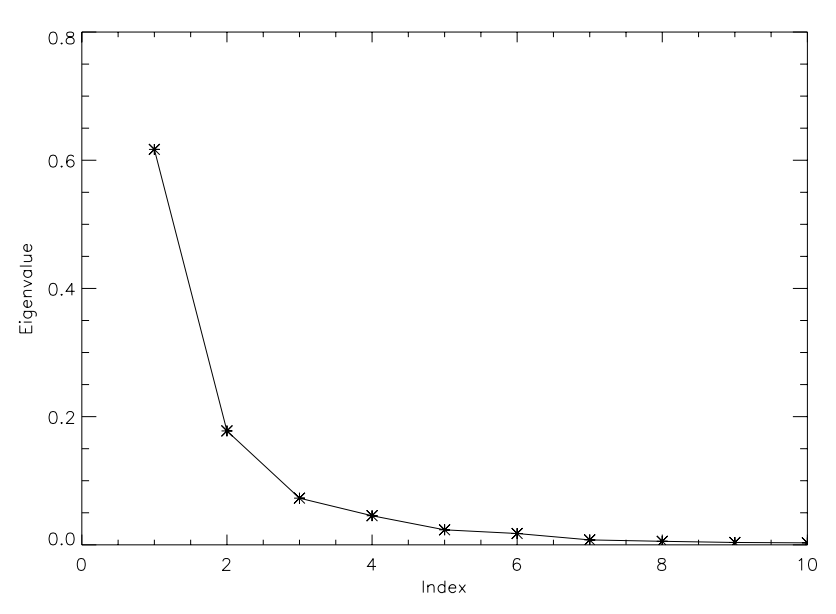

Fig. 7. The ten first singular values of the set of Stokes $Q$ profiles for the spectral region around $6301 \AA$ from the observations of August 22nd. They have been normalized to the sum of the whole set of eigenvalues. As explained in the text, the value represents the amount of information contained on the respective eigenprofile. The continuous line is for clarity purposes only

magnetic field. We also wanted to obtain a value for the noise in a magnetically sensitive line in the presence of field. We used the PCA techniques for this purpose (Rees et al. 1999) and applied them to the series of 74 images taken on August 22nd for the spectral domain around the $6301 \AA$ Fe I line. Some 14000 profiles for each one of the Stokes parameters were extracted from this series and used to create a database, from which PCA procedures derived 4 sets of eigenprofiles, one for each Stokes parameter. We also obtained the singular values associated with each one of the eigenprofiles. These singular values are an indication of the quantity of information contained in the respective eigenprofiles. For instance, Fig. 7 shows the ten first singular values obtained for Stokes $Q$. The entire set of eigenvalues represents the total information contained in the whole set of $Q$ profiles extracted from the referred data. In the figure, the eigenvalues have been normalized to the sum of all the singular values, and its interpretation is very easy. For example the first eigenprofile contains on average $60 \%$ of the information present in each profile of the set taken into consideration. In fact it can be shown that this first eigenprofile is the mean profile of the set. The second eigenprofile will account for $17 \%$, the third for $7 \%$ and so on. In total, $97 \%$ of the information contained in any particular profile can be reproduced by an adequate combination of the 10 first eigenprofiles. The form of the curve in Fig. 7 gives us also an indication of what kind of information each eigenprofile is taking into account. PCA does not make the difference between signal and noise, but it concentrates the organized patterns in the data in a few singular values: the bigger ones. To 

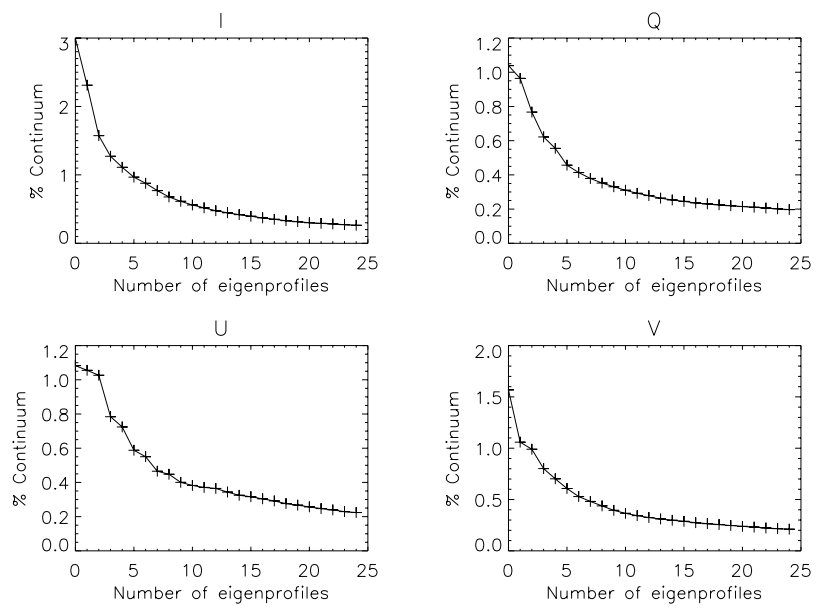

Fig. 8. Standard deviation (in $\%$ of the continuum signal) of the differences between the whole set of observed profiles and its reconstruction using a variable number of eigenprofiles. The continuous line is for clarity purposes only. The curves flatten from the moment they are reproducing noise. No more than 10 eigenprofiles suffice to take into consideration most of the information contained in the profiles

reproduce the non-organized part of the data (i.e. noise and singular physical configurations) PCA require a lot of eigenprofiles. The respective singular values (the ones with higher indices) will therefore have similar values, indicating similar amounts of information. The curve with the singular values becomes flattened when the respective eigenprofiles are reproducing noise and not signal. In that sense PCA behaves similarly to a Fourier transform, where the noise is concentrated into the high frequencies, but it is much more efficient in distinguishing it. A more detailed account of the properties of this mathematical technique can be found in the book Numerical Recipes (Press et al. 1988), or in the papers by Hansen (1992), Hansen et al. (1992) and Hansen \& Prost O'Leary (1993). For an application of this technique to solar and stellar spectral profiles, see Rees et al. (1999).

Back to our noise measurements, ten eigenprofiles are more than enough to reproduce the signal in the data discarding noise. However, to test the noise, we have reconstructed every profile present in the referred set with a combination of up to 25 eigenprofiles, and calculated what was the residual left. The results are presented in Figs. 8 and 9. Once more we see in these figures that the first eigenprofile suffices to reproduce each one of the observed profiles within 1 to $3 \%$ of the signal of the continuum. This is however not enough and between 5 and 10 eigenprofiles must be used to flatten the four standard deviation curves and drive the mean error to 0 (indicating a well centered noise). The error committed in the reconstruction without noise is of $410^{-3}$ approximately, except for Stokes $I$ which is kept at levels of $810^{-3}$ for such a small number
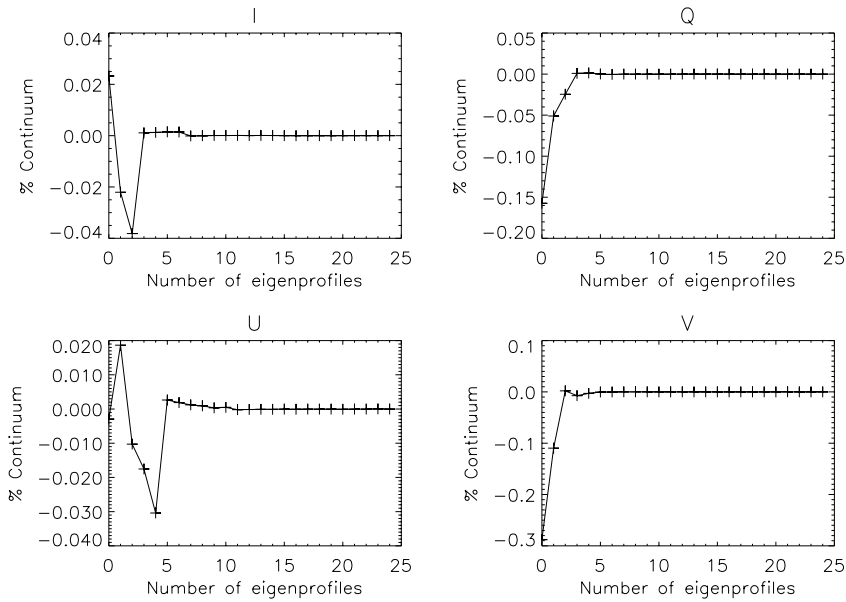

Fig. 9. Means (in $\%$ of the continuum signal) of the differences between the whole set of observed profiles and its reconstruction using a variable number of eigenprofiles. The continuous line is for clarity purposes only. The means go to zero when the residuals are just white noise. Once more (see previous figure) we see that at most 10 eigenprofiles are necessary to reproduce the information contained in the whole set of profiles

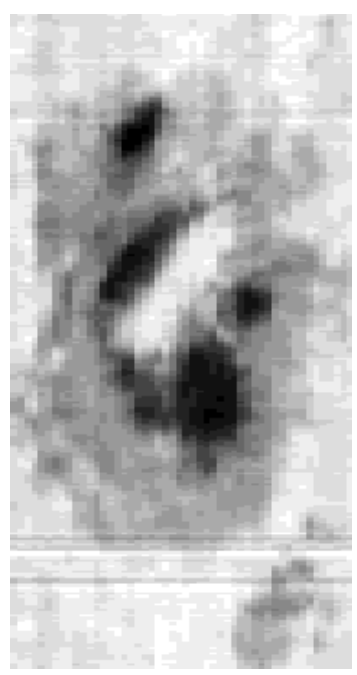

Fig. 10. Image of the continuum for NOAA-8307 on August 22 th (see the text). The image is $125 \times 243$ pixels, each one spanning $0.4^{\prime \prime}$

of eigenprofiles. These two numbers give the polarimetric noise level in the lines in the presence of signal.

The profiles considered in the series of data include all the polarization signals of the observed spot for two magnetic-sensitive lines and two telluric lines. The obtained noise values are slightly higher (except for intensity for which it is clearly bigger) than those measured in the continuum. This is not surprising, as there is less signal in the lines than in the continuum, and furthermore, the data comes from a scan over a sunspot (while the continuum measurements were done for data taken on the disk 


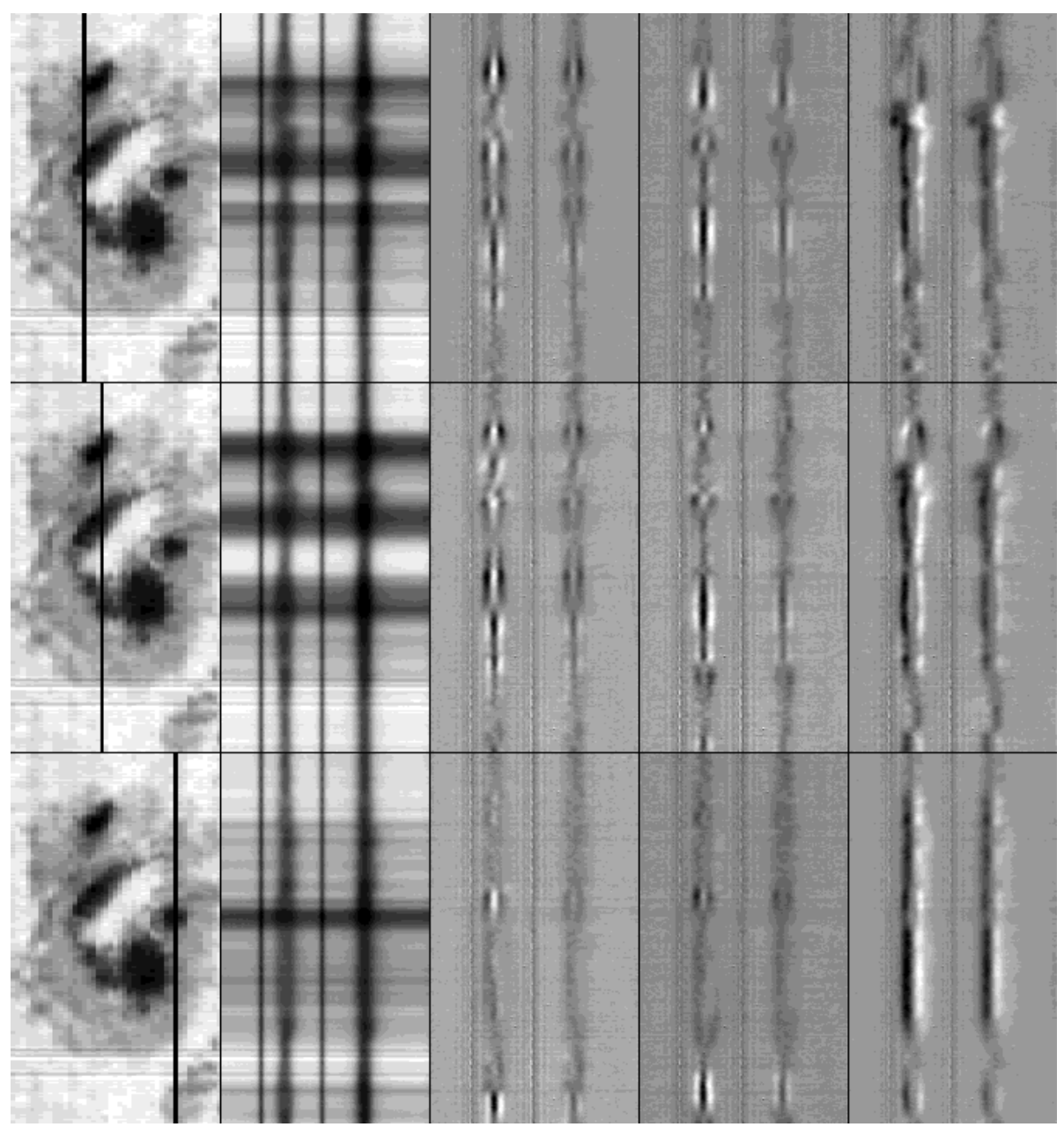

Fig. 11. The continuum image at left indicates the slit position for which the four Stokes parameters are shown $(I, Q, U$ and $V$ from left to right) for the spectral region around $6301 \AA$

center and in the quiet sun) so the photon noise is also higher. Hence the obtained result is consistent with the previous measurements.

Table 3. Noise in data

\begin{tabular}{l|r} 
Photon Noise & $2.410^{-3}$ \\
Polarimetric sensitivity in the continuum & $30^{-3}$ \\
Polarimetric sensitivity in the lines & $3-410^{-3}$
\end{tabular}

\subsection{Summary}

The three noise measurements, which we have summarized in Table 3, are consitent with each other, and we can conclude that the photon noise level was under $310^{-3}$ and that a polarimetric sensitivity of 3 to $410^{-3}$ of the intensity of the continuum was available at the moment of these observations. It is very important to note that these measurements do not take into account systematic errors.
Among these systematic errors we indicate the following:

- The observed difference between the equivalent widths for the two paths, probably due to the presence of polarized scattered light in the spectrograph;

- The errors in the correction of the spatial and temporal gains per pixel;

- The errors in the correction of the geometry between the two cameras;

- And the errors in the polarization analyser, which will induce crosstalk among the Stokes parameters.

The first one is the most conspicuous in the reduced data and must be the object of a thorough test for subsequent observations. The last one is undoubtedly present, but the actual data does not allow a calibration nor even an estimation of this effect. Without such a calibration the expected residual instrumental polarization due to stresses in the entrance window may not be removed. 


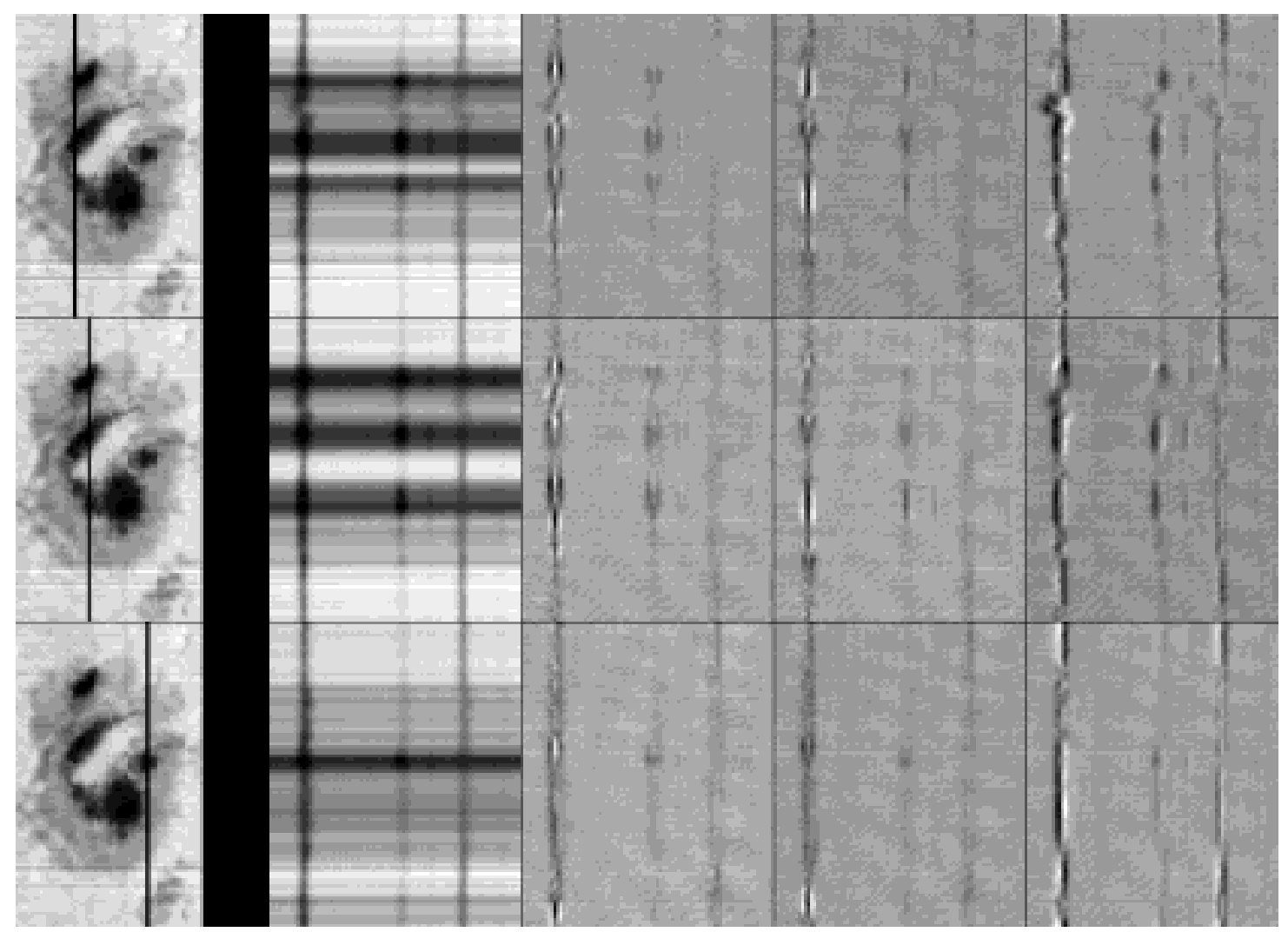

Fig. 12. The same as Fig. 11 except for the spectral region around $6149 \AA$
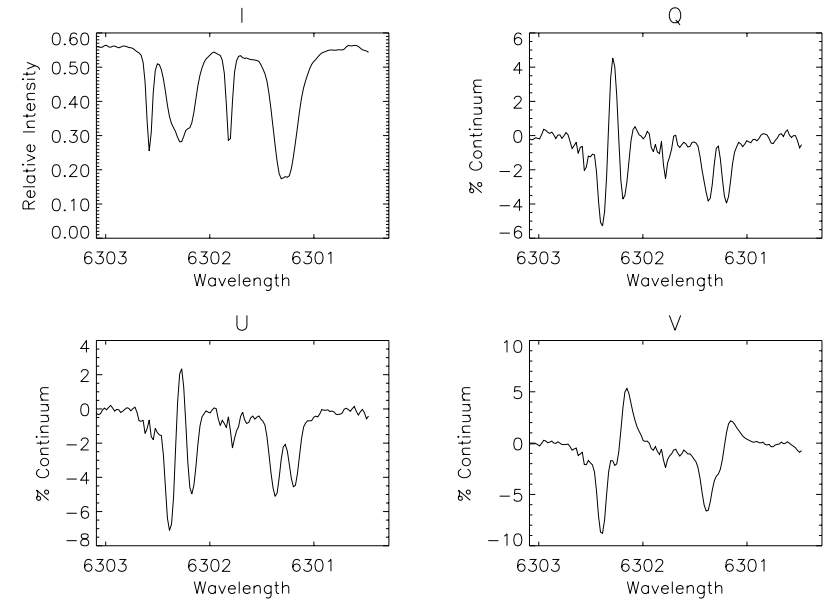

Fig. 13. The 4 Stokes parameters for a point of the first slit image in Fig. 11 at the spectral region around $6301 \AA$. Note the small inversion in the $V$ profile center, probably due to anomalous dispersion. Note also the asymmetries in the Stokes profiles; they should be compared with those in the profiles of the same slit position shown in Fig. 14

\section{Some examples}

After discussing the problems and solutions provided for this first light data set in the MTR mode of THEMIS, some examples of this data should be shown. No physical
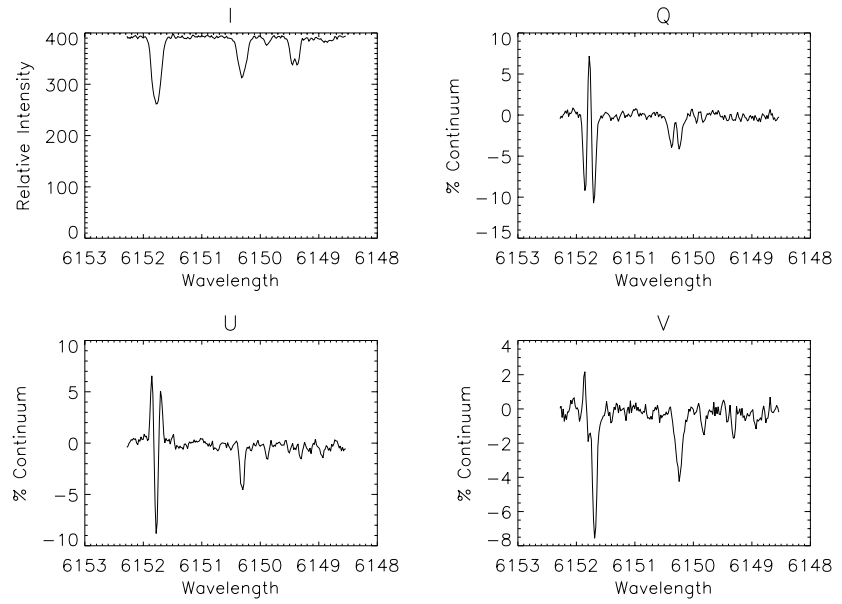

Fig. 14. Asymetries in the profiles are evident at this point of the slit extracted from the first image in Fig. 12 in the spectral region around $6149 \AA$. To be compared with the ones found in Figs. 13 and 15

interpretation has been given to the present data. They have been used to guess what is happening in the telescope and not in the sun. They are just the first step on the way to obtaining physically meaningful data in future observations. 

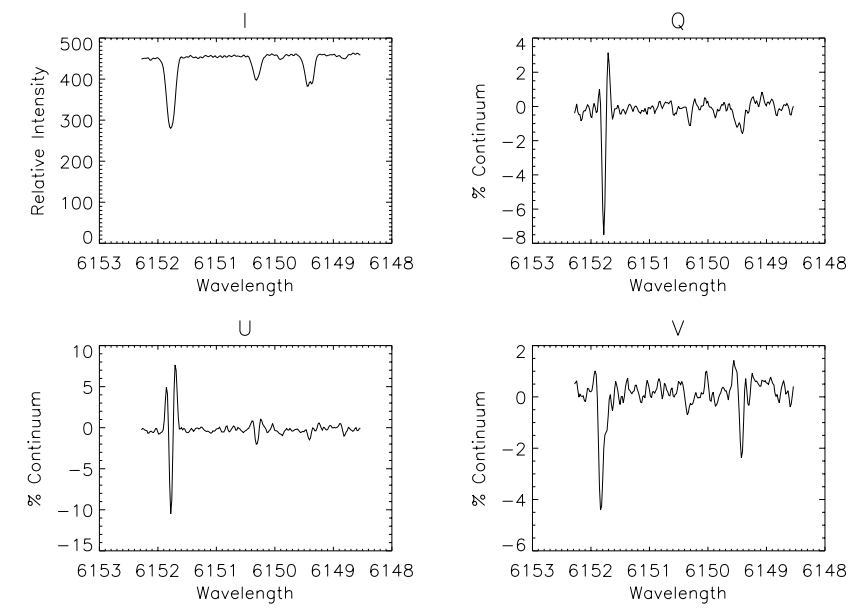

Fig. 15. Another set of Stokes profiles for the spectral region near $6149 \AA$ extracted from the same slit as previous figure. Note the inverted asymetries relative to that previous figure

The data have been selected from observations on August 22nd at 8:37 AM at the referred spot (see Fig. 10). The two plates in Figs. 11 and 12 show 3 slit positions for both the $6301 \AA$ and the $6149 \AA$ regions. For each one of the slit positions the 4 Stokes parameters are presented. As a first illustration, we have extracted from this data a point (Fig. 13) which shows an inversion in Stokes $V$ in the center of the line probably due to anomalous dispersion. The signal level is of the same order of the measured noise, we therefore conclude that, at least at first order and without further analysis, the errors introduced by the analyser and any instrumental polarization are at most comparable with the noise.

The most apparent aspect of those profiles is the presence of polarization signal for the two $\mathrm{O}_{2}$ telluric lines. This is the result of the different equivalent widths of the lines in the two orthogonally polarized paths. As a normalization has been imposed to the continuum signals, this difference translates into a difference in the depth of lines. After comparison of the images, it becomes an absorptionlike signal. This phenomenon can also be seen in the Fe I line at $6149 \AA$, which due to its atomic level configuration, lacks linear polarization signal. However, for this line the equivalent width problem and the other errors were less prominent (probably due to its larger equivalent width compared to the telluric lines) and only a small residual can be found.

Figures 14 and 15 show two sets of Stokes profiles extracted from the same image (the first slit in plate 12). The asymmetries in the Stokes $U$ profiles are evident but different in sign, indicating a clear and exclusive solar origin. Stokes $V$ has also very pronounced asymmetries which deform completely the usual antisymmetries of this profile. Comparison with the $V$ profiles at other wavelengths (as the one shown in Fig. 13, taken in a near solar point and simultaneously with the two referred profiles) excludes crosstalk between Stokes parameters as the primary origin of these strongly asymmetric $V$ profiles. Finally, in Fig. 16 we present three charts of longitudinal magnetic field as measured by the center of gravity method on the $6301 \AA, 6302 \AA$ and $6151 \AA$ Fe I lines. No attempt at calibration has been made and the grey scale is in terms of pixel length displacements. They must be seen in a qualitative way: the distribution of magnetic field corresponds to what can be expected and there is a general coherence between the images measured on three different lines. The differences between these images show the importance of observing and analysing several spectral lines simultaneously in order to obtain a more precise diagnostic of the distribution of magnetic field and its gradients in the solar photosphere.

\section{Comments and conclusions}

In this paper we have presented the first results obtained with the spectropolarimetric mode of THEMIS, based on observations made in August, 1998. The main interest of these results is that they provide a practical description of the state of the instrument. We describe the particular features of the raw data and the methods we have applied to reduce it. A particular attention has been given to the geometrical corrections. The reason for this can be described as one of the most important laws in polarimetry: the two images we compare to extract the polarization signal must be completely identical except for this polarization signal. Any other difference between them will contaminate the result. In particular, each pixel in one image must correspond to the same wavelength and spatial point in the sun as the comparison pixel in the other image. We describe the two methods which have been used for that purpose, with mainly identical results and precisions (up to several hundredths of pixel when the full profile was considered).

We have also detected some systematic errors in the instrumental setup. The most severe one was the difference in equivalent width of all the spectral lines when travelling through one or the other orthogonally polarized path. This and other errors are expected to disappear in the following campaigns, as the instrument becomes more and more tested. Apart from systematic errors, and independently of them, we have measured the noise in the data. Three different procedures have been used to estimate closely related noises. By means of statistics we have measured the photon noise and the polarimetric sensitivity on the continuum. For this last point, special attention was put into ensuring that the compared images were as identical as possible and that any residual signal could only come from noise. The Fe I line at $5576 \AA$, insensitive to magnetic field, was very useful to this aim. We have also estimated the polarimetric sensitivity in the line while scanning active regions. The presence of different polarization signals, 

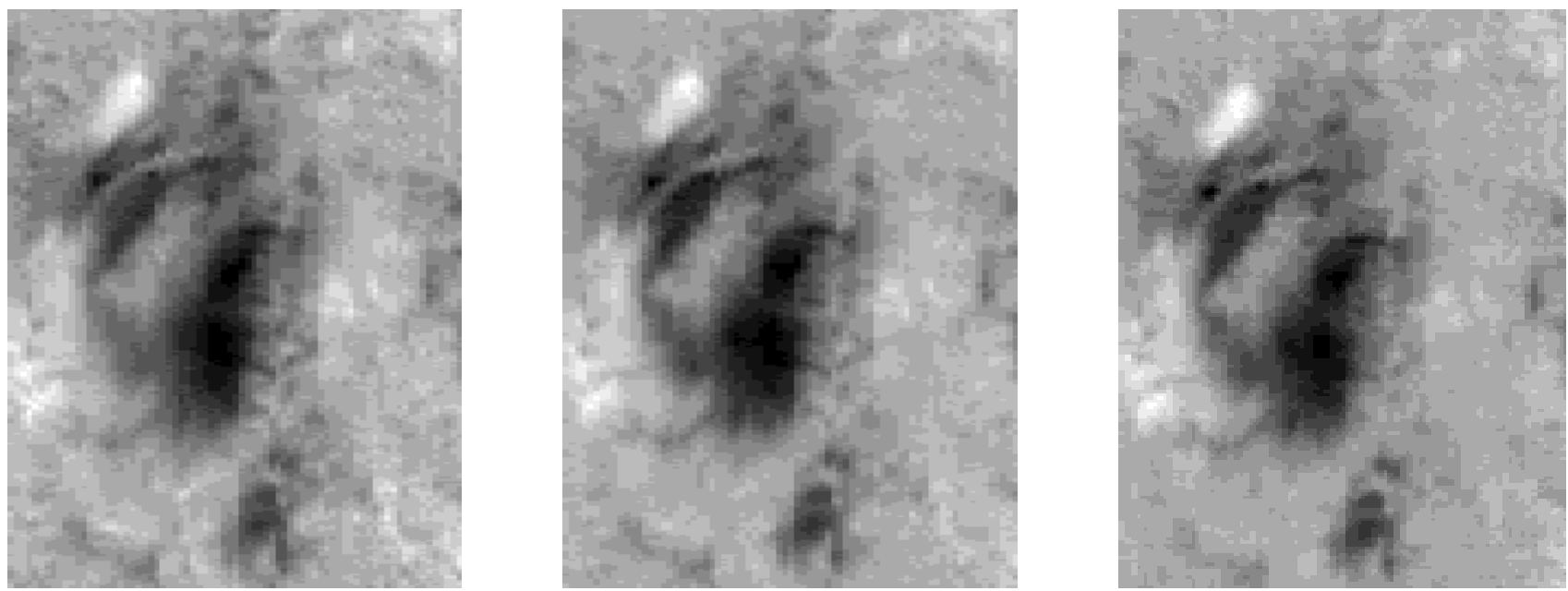

Fig. 16. Charts of longitudinal magnetic field as measured by the center of gravity method on the Fe I lines at $6301 \AA$ (left), $6302 \AA$ (center) and $6151 \AA$ (right)

in amplitude, but also in form, makes it difficult to apply plain statistics. We relied on a more powerful technique, Principal Component Analysis (PCA), to detect the noise and separate it from the signal. A brief practical description of this technique and its present use has been given; however the interested reader should refer to the appropriate literature for a thorough understanding (Press et al. 1988; Golub \& Van Loan 1993; Rees et al. 1999).

The three noise measurements are summarized in Table 3. They are coherent with each other, the highest one being that of the polarimetric sensitivity in the line, where less light is detected and for which a lot of manipulations have been done during the data reduction. In all the cases the noise is kept in a few units of $10^{-3}$ of the intensity of the continuum.

Acknowledgements. V. Bommier participated in these first spectropolarimetric observations at THEMIS and her carefully kept notebook has been of great help during the data reduction. She has also shared with the authors her experience with her own data taken almost simultaneously. We are indebted to her. Special thanks for their help during the observations are also due to THEMIS telescope operators.

\section{References}

Golub G., Van Loan C., 1993, Matrix computations (2nd ed.). The John Hopkins University Press
Hansen P.C., 1992, SIAM Rev. 34(4), 561

Hansen P.C. and D., P.O., 1993, SIAM J. Sci. Comput. 14(6), 1487

Hansen P.C., Sekii T., Shinahashi H., 1992, SIAM J. Sci. Stat. Comput. 13(5), 1142

Martínez Pillet V., Lites B.W., Skumanich A., 1997, ApJ 474, $810+$

Mein P., Rayrole J., 1985, Vistas Astron. 28, 567+

Press W., Flannery B.P., Teukolsky S.A., Vetterling W.T., 1988, Numerical Recipes: The Art of Scientific Computing. Cambridge University Press

Rayrole J., 1967, Ann. Astrophys. 30, 257

Rayrole J., 1971, IAU Symp. 43, 181+

Rayrole J., Mein P., 1993, in ASP Conf. Ser. 46: IAU Colloq. 141: The Magnetic and Velocity Fields of Solar Active Regions, pp. 170+

Rees D., Thatcher J., López Ariste A., Semel M., 1999 (to be submitted)

Semel M., 1981, A\&A 97, 75

Skumanich A., Lites B.W., Martínez Pillet V., Seagraves P., 1997, ApJS 110, 357+

Stenflo J., Keller K., 1996, Nat 382, 588

del Toro Iniesta J., Semel M., Collados M., Sánchez Almeida J., 1990, A\&A 227, 591

Westendorp Plaza C., del Toro Iniesta J.C., Ruiz Cobo B., Martínez Pillet V., Lites B.W., Skumanich A., 1997, Nat 389,47 